\title{
Variation of Molecular Alignment as a Means of Resolving Orientational Ambiguities in Protein Structures from Dipolar Couplings
}

\author{
H. M. Al-Hashimi, $* \dagger$ H. Valafar,* M. Terrell,* E. R. Zartler,* M. K. Eidsness, \\ *Complex Carbohydrate Research Center, University of Georgia, Athens, Georgia 30602; †Yale University, Chemistry Department, \\ New Haven, Connecticut 06511; and \$Chemistry Department, University of Georgia, Athens, Georgia 30602
}

Received January 24, 2000

\begin{abstract}
Residual dipolar couplings for pairs of proximate magnetic nuclei in macromolecules can easily be measured using highresolution NMR methods when the molecules are dissolved in dilute liquid crystalline media. The resulting couplings can in principle be used to constrain the relative orientation of molecular fragments in macromolecular systems to build a complete structure. However, determination of relative fragment orientations based on a single set of residual dipolar couplings is inherently hindered by the multi-valued nature of the angular dependence of the dipolar interaction. Even with unlimited dipolar data, this gives rise to a fourfold degeneracy in fragment orientations. In this Communication, we demonstrate a procedure based on an order tensor analysis that completely removes this degeneracy by combining residual dipolar coupling measurements from two alignment media. Application is demonstrated on ${ }^{15} \mathrm{~N}-{ }^{1} \mathrm{H}$ residual dipolar coupling data acquired on the protein zinc rubredoxin from Clostridium pasteurianum dissolved in two different bicelle media. $\odot 2000$ Academic Press
\end{abstract}

Key Words: NMR; order matrix; bicelle; rubredoxin; protein fold.

\section{INTRODUCTION}

The measurement of residual dipolar couplings between protein backbone nuclei can provide an alternative to NOE information in the determination of protein folds $(1-8)$. These measurements can constrain the relative orientation of molecular fragments regardless of their separation in space, a fact of considerable value when studying loosely connected protein domains or backbone segments of proteins that are separated by unassigned side chain atoms $(5,6,9)$. However, relative fragment orientations cannot be uniquely determined from a single set of residual dipolar coupling measurements, no matter how numerous the measurements $(6,9-11)$. This limitation ultimately arises from the multi-valued character of angular dependent residual dipolar coupling function,

\footnotetext{
${ }^{1}$ To whom correspondence should be addressed at Complex Carbohydrate Research Center, 220 Riverbend Road, Athens, GA 30602. E-mail: jpresteg@ccrc.uga.edu.
}

$$
\left(\frac{3 \cos ^{2} \theta-1}{2}\right)
$$

where $\theta$ is the angle between the internuclear vector and the magnetic field $(1,12)$. For a single residual dipolar coupling measurement, it is easy to see that one can only constrain the orientation of an internuclear vector into two "cones" of orientation (10). More measurements restrict solutions to intersection of cones but a single allowed solution is never found (10).

Using an order matrix analysis of multiple measurements from noncollinear vectors within a single fragment, it is easy to show that uncertainty in orientation reduces only to a fourfold degeneracy $(6,9,11-14)$. In this analysis, residual dipolar coupling measurements are used to determine the five independent elements of a symmetric and traceless $3 \times 3$ order matrix $(11,13)$. These five parameters can be reformulated in terms of an alignment of axes (three angles) for a principal order frame, and values of a principal order parameter $\left(S_{z z}\right)$, and an asymmetry parameter $\left.\left(\eta=\mid\left(S_{x x}-S_{y y}\right) / S_{z z}\right) \mid\right)(11,13)$. Because the order parameters are themselves insensitive to axis inversion, there are four possible ways to direct axis in an aligned right-handed principal axis system $(6,11,13)$. We show here that this degeneracy can be simply resolved by solving for order tensors in two different alignment media.

Bax and co-workers previously argued for the utility of measuring independent sets of residual dipolar couplings to reduce orientation degeneracy using the cone model (10), and Clore et al. implemented this idea in a simulated annealing protocol which simultaneously uses constraints from NOE data (5). In this protocol the axial and rhombic components of the alignment tensor (2 parameters) are commonly estimated from a large distribution of residual dipolar couplings $(15,16)$ and the orientation of the order tensor ( 3 parameters) is allowed to float during structure refinement (5). They demonstrated that inclusion of an additional set of residual dipolar couplings increased the precision in structure determination of the B1 domain of streptococcal protein $\mathrm{G}$ by $20-30 \%$ (5).

The potential impact of resolving orientational ambiguity is 
A Alignment 1:
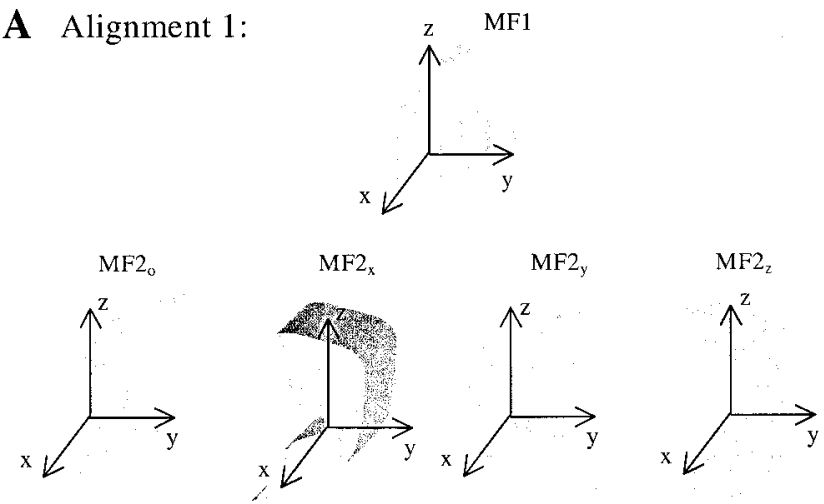

B Alignment 2:

MFI
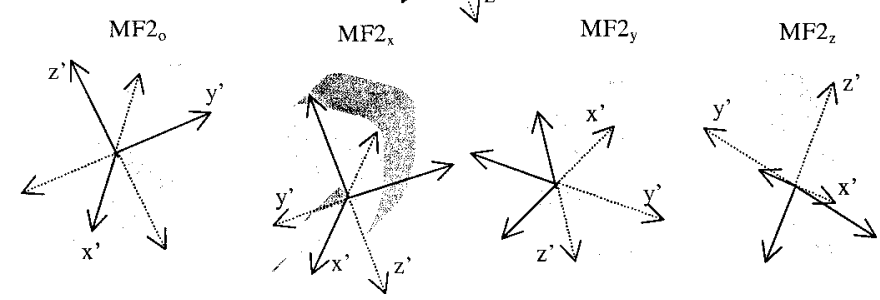

FIG. 1. Resolving orientational degeneracy using two noncoincident order tensors. (A) The four possible ways to assemble two molecular fragments using a single order tensor frame from alignment medium 1 . Note that while the molecular orientations $\mathrm{MF} 2_{\mathrm{o}}, \mathrm{MF} 2_{\mathrm{x}}, \mathrm{MF} 2_{\mathrm{y}}$, and $\mathrm{MF} 2_{\mathrm{z}}$ will always be distinguishable for nonsymmetrical molecules, the designation "o," "x," "y," and " $\mathrm{z}$ " is arbitrary. (B) The orientation of the order tensor frame $\left(x^{\prime}, y^{\prime}, z^{\prime}\right)$ using alignment medium 2 from the point of view of the molecular orientations shown in Fig. 1A. Only one of the four possible orientations $\left(M F 2_{x}\right.$ shaded in black) has a coincident order tensor frame with MF1.

even greater for an order tensor approach to structure determination because this approach can be implemented with near total dependence on orientational data as opposed to NOE data (13). Here, structure determination is based on aligning successive, fragment-centered order tensor frames. While 2 fragments can be assembled with a 4-fold degeneracy, degeneracy rises to $4^{n-1}$ when $n$ fragments are to be assembled $(11,12)$. So far, degeneracies resulted from using the order tensor approach have been resolved by either using modeling studies or inclusion of a minimal number of useful NOE constraints after the fact $(6,9,14)$. Here we extend previously presented order tensor analysis strategy to resolve this degeneracy issue by using two sets of independent residual dipolar couplings measured in media giving two noncoincident order tensors.

This order tensor approach is illustrated in Fig. 1, where we have cut a molecule into two molecular fragments (MF1 and MF2) and determined an order tensor and principal alignment frame ( $x, y$, and $z$ axes) independently using residual dipolar data from each fragment $(11,13)$. Since we are not interested in average molecular orientation of the entire molecule, we can arbitrarily choose one of the four allowed frames determined in the first medium as a reference structure (top of Fig. 1A). We can align each of the possible frames for MF2 in this medium with the reference frame by rotating the fragments (bottom of Fig. 1A). This gives four possible ways to assemble MF1 and MF2 into a structural model and only one of these possibilities corresponds to the correct structure.

In order to determine the correct orientation from the four possibilities shown in Fig. 1A, we collect a second set of residual dipolar coupling data in a different alignment medium (10) (alignment 2) and again determine principal alignment frames as viewed from each fragment (Fig. 1B). We orient MF1 to superimpose with the reference orientation of MF1 of Fig. 1A top (Fig. 1B top), and solve for the possible principal alignment frames (all possible directions for $x^{\prime}, y^{\prime}$, and $z^{\prime}$ are shown). We then orient MF2 to superimpose with each of the four possibilities shown in Fig. 1A and solve for the alignment frame orientation in each case (Fig. 1B bottom). Only in one case, the second orientation for MF2, does the alignment frame coincide with that for MF1. Hence, the combination of MF1 and MF2 in this orientation will correspond to the correct structure.

Experimentally, this approach assumes an ability to modulate the order tensor governing partial alignment and an ability to do this in a way that doesn't simply change the magnitude or asymmetry, of alignment, but changes the order tensor orientation relative to all three principal directions. These changes in order tensor properties have previously been reported using different aligning reagents (17), changing $\mathrm{pH}$ in the presence of His-Tag on the protein (10), and by doping bicelle media with charged lipids $(10,18)$. We use the latter approach for modulating order tensor properties.

\section{RESULTS}

The experimental feasibility of the approach presented above was tested using backbone ${ }^{15} \mathrm{~N}-{ }^{1} \mathrm{H}$ residual dipolar coupling data measured in zinc rubredoxin from Clostridium pasteurianum $(\mathrm{CpZnRd})$ dissolved in two bicelle media. ${ }^{15} \mathrm{~N}$ enriched $\mathrm{CpZnRd}$ was expressed and purified as previously described (19). Bicelle media composed of dimyristoyl-phosphatidylcholine (DMPC) and dihexanoyl-phosphatidylcholine (DHPC) at 3:1 (DMPC:DHPC) molar ratios were also prepared as previously described $(3,6)$. Two NMR samples were made. Both samples contained 10\% (w/v) bicelles, $2 \mathrm{mM} \mathrm{CpZnRd,}$ $100 \mathrm{mM}$ phosphate buffer, and $150 \mathrm{mM}$ sodium sulfate. One sample was doped with negatively charged sodium dodecyl sulfate (SDS) (4\% relative to DMPC) while the second sample was doped with positively charged cetyltrimethylammonium bromide (CTAB) (3\% relative to DMPC). Doping bicelles with charged lipids has also been previously described, for enhancing bicelle stability (18), for reducing macromolecule interactions with bicelles (18), and for obtaining a new independent 


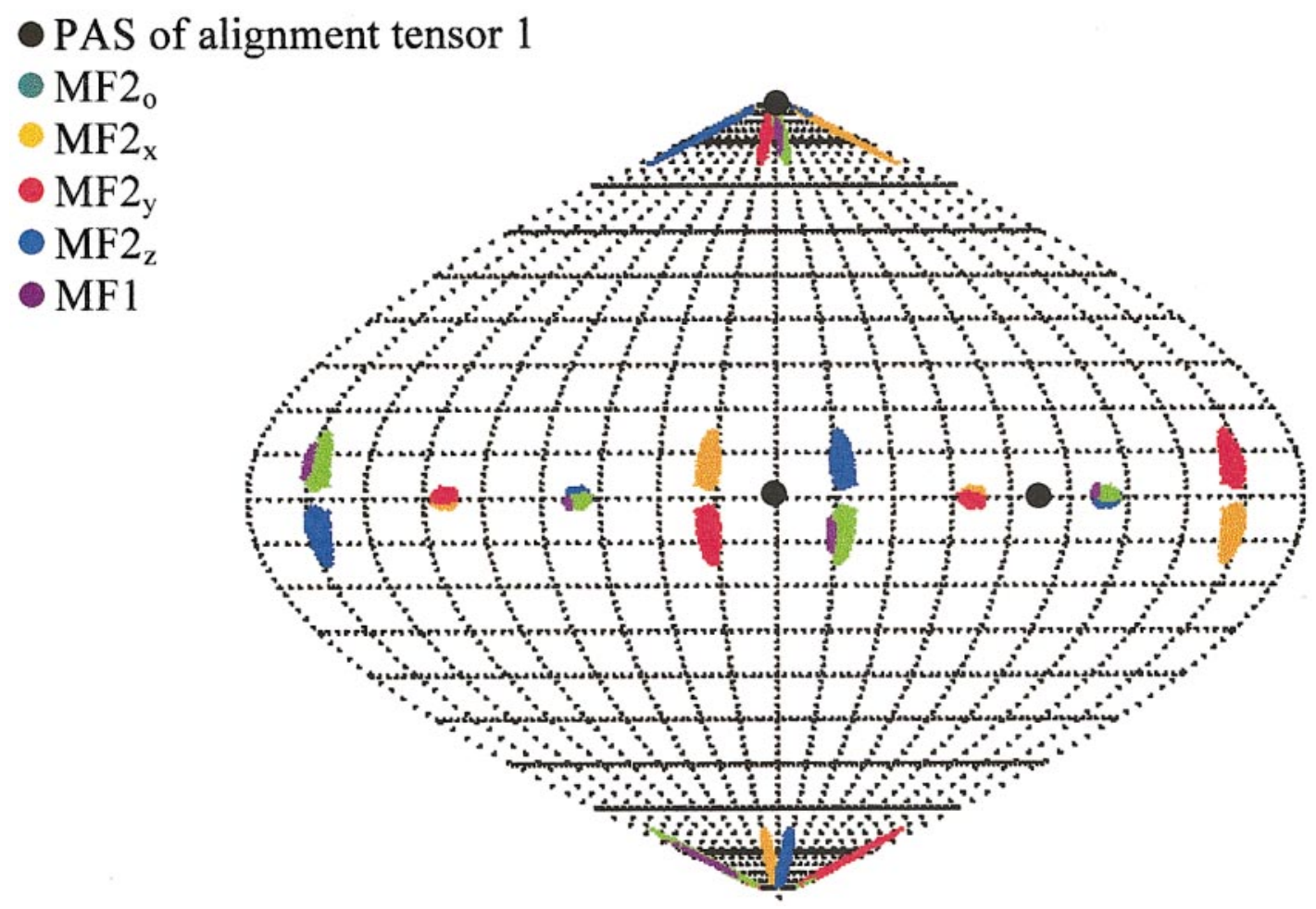

FIG. 2. A Sauson-Flamsteed projection map depicting the orientational solutions determined for two molecular fragments in CpZnRd (MF1 and MF2 see text) using residual dipolar couplings measured in a CTAB doped bicelle medium. All solutions are depicted relative to the principal axis system (PAS) determined for an SDS doped bicelle medium (shown as black circles). Orientational solutions were obtained using a previously described program called "ORDERTEN_SVD" (11) (available at the Web address: http://tesla.ccrc.uga.edu) using 100,000 reiterations. Note that only one set of solutions (MF2 ${ }_{0}$ ) of MF2 overlaps with orientational solutions for MF1 in all three principal directions.

residual dipolar coupling data $(10) .{ }^{15} \mathrm{~N}-{ }^{1} \mathrm{H}$ splittings were measured using a coupling enhanced variation of an indirectly coupled HSQC, where couplings are allowed to evolve for twice the time in the indirect dimension $\left(2 t_{1}\right)$ compared to chemical shift $\left(t_{1}\right)(20)$. This experiment yields a $2 \mathrm{D}{ }^{15} \mathrm{~N}-{ }^{1} \mathrm{H}$ correlation spectrum where contours in the indirect dimension are split by twice the value of ${ }^{15} \mathrm{~N}-{ }^{1} \mathrm{H}$ couplings $(20) .{ }^{15} \mathrm{~N}-{ }^{1} \mathrm{H}$ splittings were measured as peak separations from 1D slices along the ${ }^{15} \mathrm{~N}$ dimension. Errors in splittings determined from the rmsd between measured values, from pairs of identically acquired spectra, indicate a uniform precision of measurement of approximately $1 \mathrm{~Hz}$. Residual dipolar coupling data $(D)$ were calculated as the difference between splittings measured in the absence $(J)$ and presence of bicelle aligning reagent $(J+D)$. A final error of $2 \mathrm{~Hz}$ was used in the analysis of residual dipolar couplings.

Data were then clustered into two sets representing distinct fragments of the $\mathrm{CpZnRd}$ molecule. One fragment contained residues 1-14 and 37-53 which are close to the metal center (MF1) while the other fragment contained all remaining residues 15-36 (MF2). The five order tensor parameters for MF1 and MF2 were independently computed for the SDS medium and for the CTAB medium using ${ }^{15} \mathrm{~N}-{ }^{1} \mathrm{H}$ residual dipolar couplings (19 data in MF1 and 8 data in MF2). Internuclear

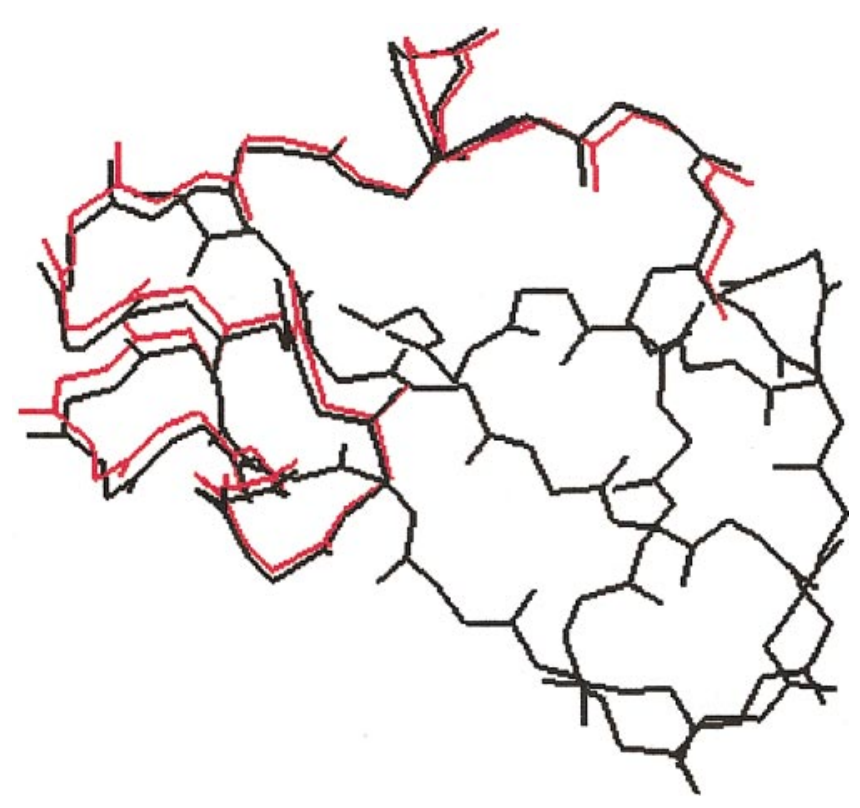

FIG. 3. Comparison of the reassembled CpZnRd molecule determined using residual dipolar couplings from two different media with the X-ray structure (PDB 1IRN) (21). Molecular fragment 1 (MF1, shown in black) is superimposed in the two structures and the relative orientation of MF2 is shown in black (X-ray) and red (residual dipolar couplings) (21). The deviation in relative orientation does not exceed $5^{\circ}$. 
coordinates from an X-ray structure (PDB 1IRN) (21) were used along with a previously described program ORDERTEN_SVD to carry out the calculation (11). Rather than using graphical depictions of the molecular fragments with superimposed axes, we plot experimentally determined axis-directions as clusters of points on a projection of a globe called a Sauson-Flamsteed plot (Fig. 2) $(11,14)$. This procedure follows the depictions shown in Fig. 1. We chose one orientation for MF1 as a reference frame for the data in the SDS doped bicelle, such that the $z$ axis is at the apex of the Sauson-Flamsteed plot and the $x$ axis extends from the center (black dots). Four orientations for $\mathrm{MF} 2\left(\mathrm{MF} 2_{0}, \mathrm{MF}_{\mathrm{x}}, \mathrm{MF}_{2}\right.$, and $\mathrm{MF}_{\mathrm{z}}$ ) that are consistent with the SDS data and that yield coincident order tensor frames with MF1 were generated by $180^{\circ}$ rotations about the principal $x, y$, and $z$ axes (Fig. 1A). Solutions for order tensor frames determined in the CTAB doped bicelle are shown in various colors. We show solutions for the reference MF1 in violet. Note that orientations of SDS and CTAB frames differ by $\approx 20^{\circ}$ in the $x$ and $y$ direction and $\approx 10^{\circ}$ in the $z$ direction; the two order tensors are noncoincident. The five elements of the order tensor for the CTAB medium were determined from the point of view of MF2 using the four orientations $M F 2_{0}, \mathrm{MF}_{2}, \mathrm{MF} 2_{\mathrm{y}}$, and $\mathrm{MF} 2_{\mathrm{z}}$ as input coordinates. The four sets of axis orientations are shown in Fig. 2. Note that none of principal axes determined for $M F 2_{x}$ (orange spots) or $\mathrm{MF}_{\mathrm{y}}$ (red spots) overlap with the principal axes determined for $\mathrm{MF}_{1}$ (violet spots). Only one of the principal axes ( $z$ axis shown in blue spots) of MF $2_{z}$ overlaps a principal axis of MF1 (violet spots). A consistent orientation must result in overlap of orientational solutions about all three principal axes. Therefore, none of these three orientations of MF2 relative to MF1 are consistent with residual dipolar couplings measured in bicelles doped with CTAB. The orientational solutions of MF2 (green spots) do overlap with those of MF1 in all three principal axes directions. This orientation of MF2 relative to MF1 is also in agreement with the X-ray structure orientation (PDB 1IRN) (21). Figure 3 shows both the intact rubredoxin and reassembled rubredoxin using the center of the green cluster as axis definitions for fragment 2 . It is clear that the correct geometry is selected by this analysis.

\section{DISCUSSION}

Thus far, we have demonstrated a direct approach for unambiguously determining the relative orientations of known molecular fragments using residual dipolar couplings from aligning reagents that yield noncoincident order tensors. The manipulation of bicelle systems by doping with charged lipids provides a convenient way for obtaining noncoincident order tensors, though other methods have also been proposed (10). It is important to note that the inherent resolution in discriminating between the various orientational solutions using this approach will depend on the level of noncoincidence between the two order tensors. Even though noncoincidence in the $z$ direc- tion is only on the order of $10^{\circ}$, resolution proved sufficient to discriminate between all four possible orientations. One would like to have larger differences. The high ionic strength used for stabilizing the CpZnRd sample may have contributed to the small differences by attenuating the electrostatic interactions responsible for changing partial alignment upon addition of charged lipids (18). It is possible to have large overall differences with an accidental coincidence of one axis. However, even in this case, one can still reduce the orientational degeneracy from four to two. This twofold degeneracy would remain because a $180^{\circ}$ rotation about the coincident principal axis would always yield an indistinguishable axis system.

The resolution in discriminating between orientational solutions will also depend on how well determined the order tensor orientations are. This will depend on the asymmetry parameter, $\eta$, the number of independent residual dipolar couplings measured for each fragment, and the precision of measurement. While higher asymmetry values are desired for uniquely determining the $S_{x x}$ and $S_{y y}$ orientations, we are able to successfully apply this approach with a moderately high asymmetry parameter for the CTAB alignment $(\eta=0.69)$ and not very well determined orientation for $S_{x x}$ and $S_{y y}$ (Fig. 2). In addition, we used $19{ }^{15} \mathrm{~N}-{ }^{1} \mathrm{H}$ residual dipolar coupling data from one of the molecular fragments (MF1) and only $8{ }^{15} \mathrm{~N}-{ }^{1} \mathrm{H}$ residual dipolar coupling data from the second molecular fragment (MF2). While eight is a relatively small number of residual dipolar coupling data, we were fortunate to have a broad distribution in directions making the ${ }^{15} \mathrm{~N}-{ }^{1} \mathrm{H}$ interaction vectors quite independent.

Our choice of molecular fragment was quite arbitrary. For a real situation the choice is more likely to be a secondary structural element. Secondary structural elements are an attractive choice for molecular fragments in proteins since their local geometry can be inferred from backbone NOE and ${ }^{3} J$ coupling data without the necessity to collect side chain data $(8,22)$. This level of independent interaction vectors will be reduced when molecular fragments are regular secondary structural elements such as $\alpha$-helices and $\beta$-sheets, where many ${ }^{15} \mathrm{~N}-{ }^{1} \mathrm{H}$ internuclear vectors are close to parallel (12). Supplementing ${ }^{15} \mathrm{~N}-{ }^{1} \mathrm{H}$ data with ${ }^{1} \mathrm{H}-{ }^{1} \mathrm{H}$ or ${ }^{13} \mathrm{C}-{ }^{1} \mathrm{H}$ residual dipolar coupling data would resolve limitations imposed by these regular structures and allow a broader application of this approach $(16,23-$ 25). Generating such data, and resolving the orientational degeneracy in orientation from residual dipolar couplings, is an important step toward rapidly determining protein backbone structures for unknown proteins.

\section{ACKNOWLEDGMENTS}

The authors thank Dr. Joel R. Tolman for many stimulating discussions. This work was supported by a grant from the National Science Foundation, MCB-9726341. 


\section{REFERENCES}

1. E. W. Bastian, C. Maclean, P. C. M. Van Zilj, and A. A. Bothner-by, High-resolution NMR of liquids and gases: Effects of magneticfield-induced molecular alignment, Ann. Rep. NMR Spectrosc. 19, 35-77 (1987).

2. J . R. Tolman, J . M. Flanagan, M. A. Kennedy, and J . H. Prestegard, Nuclear magnetic dipole interactions in field-oriented proteins Information for structure determination in solution, Proc. Natl. Acad. Sci. U.S.A. 92, 9279-9283 (1995).

3. N. Tjandra and A. Bax, Direct measurement of distances and angles in biomolecules by NMR in a dilute liquid crystalline medium, Science 278, 1697-1697 (1997).

4. J. H. Prestegard, New techniques in structural NMR - anisotropic interactions, Nat. Struct. Biol. 5, 517-522 (1998).

5. G. M. Clore, M. R. Starich, C. A. Bewley, M. L. Cai, and J. Kuszewski, Impact of residual dipolar couplings on the accuracy of NMR structures determined from a minimal number of NOE restraints, J. Am. Chem. Soc. 121, 6513-6514 (1999).

6. M. W. F. Fischer, J . A. Losonczi, J . L. Weaver, and J . H. Prestegard, Domain orientation and dynamics in multidomain proteins from residual dipolar couplings, Biochemistry 38, 9013-9022 (1999).

7. A. Annila, H. Aitio, E. Thulin, and T. Drakenberg, Recognition of protein folds via dipolar couplings, J. Biomol. NMR 14, 223-230 (1999).

8. H. Aitio, A. Annila, S. Heikkinen, E. Thulin, T. Drakenberg, and I. Kipelainen, NMR assignments, secondary structure, and global fold of calerythrin, an EF-hand calcium-binding protein from Saccharopolyspora erythraea, Protein Sci. 8, 2580-2588 (1999).

9. P. J. Bolon, H. M. Al-Hashimi, and J. H. Prestegard, Residual dipolar coupling derived orientational constraints on ligand geometry in a 53 kDa protein-ligand complex, J. Mol. Biol. 293, 107-115 (1999).

10. B. E. Ramirez and A. Bax, Modulation of the alignment tensor of macromolecules dissolved in a dilute liquid crystalline medium, J. Am. Chem. Soc. 120, 9106-9107 (1998).

11. J. A. Losonczi, M. Andrec, M. W. F. Fischer, and J . H. Prestegard, Order matrix analysis of residual dipolar couplings using singular value decomposition, J. Magn. Reson. 138, 334 -342 (1999).

12. J . H. Prestegard, J . R. Tolman, H. M. Al-Hashimi, and M. Andrec, in "Modern Techniques in Protein NMR" (N. R. Krishna and L. J. Berliner, Eds.). Plenum, New York, 1998.

13. A. Saupe, Recent results in the field of liquid crystals, Angew. Chem., Int. Ed. Engl. (1968).
14. J. A. Losonczi and J. H. Prestegard, Nuclear magnetic resonance characterization of the myristoylated, $\mathrm{N}$-terminal fragment of ADP. ribosylation factor 1 in a magnetically oriented membrane array, Biochemistry 37, 706-716 (1998).

15. G. M. Clore, A. M. Gronenborn, and N. Tjandra, Direct structure refinement against residual dipolar couplings in the presence of rhombicity of unknown magnitude, J. Magn. Reson. 131, 159-162 (1998)

16. G. M. Clore, A. M. Gronenborn, and A. Bar, A robust method for determining the magnitude of the fully asymmetric alignment tensor of oriented macromolecules in the absence of structural information, J. Magn. Reson. 133, 216-221 (1998).

17. J. Sass, F. Cordier, A. Hoffmann, A. Cousin, J. G. Omichinski, H. Lowen, and S. Grzesiek, Purple membrane induced alignment of biological macromolecules in the magnetic field, J. Am. Chem. Soc. 121, 2047-2055 (1999).

18. J . A. Losonczi and J. H. Prestegard, Improved dilute bicelle solutions for high-resolution NMR of biological macromolecules, J . Biomol. NMR 12, 447-451 (1998).

19. K. A. Richie, Q. Teng, C. J. Elkin, and D. M. Kurtz, 2D H-1 and 3D H-1-N-15 NMR of zinc-rubredoxins: Contributions of the betasheet to thermostability, Protein Science 5, 883- 894 (1996).

20. J. R. Tolman and J. H. Prestegard, Measurement of amide N-15$\mathrm{H}-1$ one-bond couplings in proteins using accordion heteronuclear-shift-correlation experiments, J. Magn. Reson. Series B 112, 269-274 (1996).

21. Z. Dauter, K. S. Wilson, L. C. Sieker, J. M. Moulis, and J. Meyer, Zinc- and iron-rubredoxins from Clostridium pasteurianum at atomic resolution: A high-precision model of a ZnS4 coordination unit in a protein, Proc. Natl. Acad. Sci. U.S.A. 93, 8836-8840 (1996)

22. G. M. Clore and A. M. Gronenborn, New methods of structure refinement for macromolecular structure determination by NMR, Proc. Natl. Acad. Sci. U.S.A. 95, 5891-5898 (1998).

23. P. J . Bolon and J. H. Prestegard, COSY cross-peaks from $\mathrm{H}-1-\mathrm{H}-1$ dipolar couplings in NMR spectra of field oriented oligosaccharides, J. Am. Chem. Soc. 120, 9366 -9367 (1998).

24. F. Tian, P. J. Bolon, and J. H. Prestegard, Intensity-based measurement of homonuclear residual dipolar couplings from CTCOSY, J. Am. Chem. Soc. 121, 7712-7713 (1999).

25. N. Tjandra and A. Bax, Measurement of dipolar contributions to (1)] (CH) splittings from magnetic-field dependence of J modulation in two- dimensional NMR spectra, J. Magn. Reson. 124, 512-515 (1997). 